Pure and Applied Mathematics Quarterly

Volume 3, Number 1

(Special Issue: In honor of

Robert MacPherson, Part 3 of 3 )

$283-306,2007$

\title{
Steenrod Operations, Degree Formulas and Algebraic Cobordism
}

\author{
Marc Levine \\ To Bob MacPherson on the occasion of his 60th birthday
}

\section{INTRODUCTION}

Relying on Brosnan's theory of Steenrod operations [1], Merkurjev [10] has given a wide-ranging construction of characteristic classes with values in the Chow ring, satisfying so-called degree formulas. In this paper, we give what we view as a somewhat more conceptual treatment of both Brosnan's Steenrod operations and Merkurjev's degree formulas, relying on our theory of algebraic cobordism [7] (see also the earlier preprints $[8,9]$ ). As algebraic cobordism requires resolution of singularities, our approach is limited to characteristic zero.

Briefly, the outline of our construction is as follows: Milnor [11] noted that the dual of the reduced mod- $p$ Steenrod algebra in topology is closely related to the endomorphism algebra of the additive formal group law over $\mathbb{F}_{p}$. Algebraic cobordism also has a close connection to formal group laws: algebraic cobordism is the universal oriented Borel-Moore homology theory on $\mathbf{S c h}_{k}$, to each oriented Borel-Moore homology theory $A$ on $\mathbf{S} \mathbf{c h} k$ is associated a formal group law $F_{A}(u, v) \in A_{*}(k)[[u, v]]$, and the formal group law for algebraic cobordism is the universal one. In addition, the theory given by the Chow groups of cycles modulo rational equivalence is the universal theory with additive formal group law. Relying on a twisting construction, this yields that each automorphism $\tau$ of the additive formal group law over a graded $\mathbb{F}_{p}$-algebra $R$ yields a natural transformation of the theory $\mathrm{CH}_{*} \otimes R$ to the "twisted" theory $\mathrm{CH}_{*} \otimes R^{(\tau)}$; the

Received November 11, 2005.

1991 Mathematics Subject Classification.Primary 14C25; Secondary 55P42, 18F20, 14F42.

The author gratefully acknowledges the support of the Humboldt Foundation through the Wolfgang Paul Program, and support of the NSF via grants DMS 0140445 and DMS-0457195 
mod- $p$ Steenrod operations are then given as the coefficients of the transformation resulting from the automorphism,

$$
t \mapsto t+\sum_{n \geq 1} b_{n} t^{p^{n}}
$$

over the polynomial algebra $\mathbb{F}_{p}\left[b_{1}, b_{2}, \ldots\right], \operatorname{deg}\left(b_{n}\right)=p^{n}-1$. One gets for free the naturality of the Steenrod operations with respect to projective push-forward and a Riemann-Roch formula for the behavior under pull-back by an l.c.i. morphism, as well as a product formula for the total Steenrod operation, analogous to the classical Cartan relation.

For the degree formulas, we consider a canonical integral lifting of the mod- $p$ Steenrod operations, yielding a natural transformation of theories

$$
S^{(p)}: \Omega_{*} \rightarrow \mathrm{CH}_{*}\left[b_{1}, b_{2}, \ldots\right]^{(b)},
$$

$S^{(p)}=\sum_{R} S_{R}^{(p)} b^{R}$. The naturality of the Steenrod operations with respect to push-forward yield a divisibility property of the $S_{R}^{(p)}$, giving interesting $\mathbb{Z}$-valued characteristic classes $s_{R}:=S_{R}^{(p)} / p$ on smooth projective $k$-schemes, for each index $R \neq 0$. The product formula for $S^{(p)}$, combined with the generalized degree formula for algebraic cobordism, yields the degree formulas of Merkurjev for the classes $s_{R}$.

I would like to thank the referee for his commments and suggestions and Lizhen Ji for organizing this volume. I would also like to extend my heartfelt thanks to Bob MacPherson for giving mathematical inspiration and support over many years.

\section{ORIENTED THEORIES}

Let $\mathbf{S c h}_{k}$ denote the category of separated schemes of finite type over $k$. We recall the setting of an oriented Borel-Moore theory from [7, Definition 5.1.3]; we fix a field $k$ and let pt $=\operatorname{Spec} k$. For a full subcategory $\mathcal{V}$ of $\mathbf{S c h}_{k}$, we let $\mathcal{V}^{\prime}$ denote the category with the same objects as $\mathcal{V}$, but with morphisms the projective morphisms. For us, an l.c.i. morphism is a morphism $f: Y \rightarrow X$ in $\mathbf{S c h}_{k}$ that admits a factorization $f=p \circ i$, with $i: Y \rightarrow P$ a regular embedding in $\mathbf{S c h}_{k}$ and $p: P \rightarrow X$ a smooth, quasi-projective morphism over $k$.

Given a rank $n$ locally free sheaf $\mathcal{E}$ on $X$, let $q: \mathbb{P}(\mathcal{E}) \rightarrow X$ denote the projective bundle of rank one quotients of $\mathcal{E}$, with tautological quotient invertible sheaf $q^{*} \mathcal{E} \rightarrow \mathcal{O}(1)_{\mathcal{E}}$. We let $O(1)_{\mathcal{E}}$ denote the line bundle on $\mathbb{P}(\mathcal{E})$ with sheaf of sections $\mathcal{O}(1)_{\mathcal{E}}$ 
Two morphisms $f: X \rightarrow Z, g: Y \rightarrow Z$ in $\mathbf{S c h}_{k}$ are called Tor-independent if for each triple of points $x \in X, y \in Y, z \in Z$ with $f(x)=g(y)=z$,

$$
\operatorname{Tor}_{p}^{\mathcal{O}_{Z, z}}\left(\mathcal{O}_{X, x}, \mathcal{O}_{Y, y}\right)=0
$$

for $p>0$.

We call a functor $F: \mathbf{S c h}_{k}^{\prime} \rightarrow \mathbf{A} \mathbf{b}_{*}$ additive if $F(\emptyset)=0$ and the canonical map $F(X) \oplus F(Y) \rightarrow F(X \coprod Y)$ is an isomorphism for all $X, Y$ in $\mathcal{V}$.

Definition 2.1. An oriented Borel-Moore homology theory $A$ on $\mathbf{S} \mathbf{c h}$ is given by

(D1). An additive functor

$$
A_{*}: \mathbf{S c h}_{k}^{\prime} \rightarrow \mathbf{A} \mathbf{b}_{*}, X \mapsto A_{*}(X) .
$$

(D2). For each l.c.i. morphism $f: Y \rightarrow X$ in $\mathbf{S c h}_{k}$ of relative dimension $d$, a homomorphism of graded groups

$$
f^{*}: A_{*}(X) \rightarrow A_{*+d}(Y) \text {. }
$$

(D3). An element $1 \in A_{0}(\mathrm{pt})$ and, for each pair $(X, Y)$ in $\mathbf{S c h}_{k}$, a bilinear graded pairing:

$$
\begin{aligned}
A_{*}(X) \otimes A_{*}(Y) & \rightarrow A_{*}\left(X \times_{k} Y\right) \\
u \otimes v & \mapsto u \times v,
\end{aligned}
$$

called the external product, which is associative, commutative and admits 1 as unit element.

These satisfy

(BM1). One has $I d_{X}^{*}=I d_{A_{*}(X)}$ for any $X \in \mathbf{S c h}_{k}$. Moreover, given composable l.c.i. morphisms $f: Y \rightarrow X$ and $g: Z \rightarrow Y$ in $\mathbf{S c h}_{k}$ of pure relative dimension, one has $(f \circ g)^{*}=g^{*} \circ f^{*}$.

(BM2). Let $f: X \rightarrow Z$ and $g: Y \rightarrow Z$ be morphisms in $\mathbf{S c h}_{k}$. Suppose that $f$ and $g$ are Tor-independent, that $f$ is projective and that $g$ is an l.c.i. morphism, giving the cartesian square

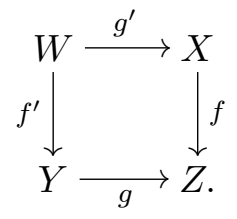

Note that $f^{\prime}$ is projective and $g^{\prime}$ is an l.c.i. morphism. Then $g^{*} f_{*}=f_{*}^{\prime} g^{\prime *}$. (BM3). Let $f: X^{\prime} \rightarrow X$ in $\mathbf{S c h}_{k}$ and $g: Y^{\prime} \rightarrow Y$ be morphisms in $\mathbf{S c h}_{k}$. If $f$ and $g$ are projective, then for $u^{\prime} \in A_{*}\left(X^{\prime}\right)$ and $v^{\prime} \in A_{*}\left(Y^{\prime}\right)$ one has

$$
(f \times g)_{*}\left(u^{\prime} \times v^{\prime}\right)=f_{*}\left(u^{\prime}\right) \times g_{*}\left(v^{\prime}\right) .
$$


If $f$ and $g$ are l.c.i. morphisms, then for $u \in A_{*}(X)$ and $v \in A_{*}(Y)$ one has

$$
(f \times g)^{*}(u \times v)=f^{*}(u) \times g^{*}(v)
$$

(PB). For $L \rightarrow Y$ a line bundle on $Y \in \mathbf{S c h}_{k}$ with zero-section $s: Y \rightarrow L$, define the operator

$$
\tilde{c}_{1}(L): A_{*}(Y) \rightarrow A_{*-1}(Y)
$$

by $\tilde{c}_{1}(L)(\eta)=s^{*}\left(s_{*}(\eta)\right)$. Let $\mathcal{E}$ be a rank $n+1$ locally free coherent sheaf on $X \in \mathbf{S c h}_{k}$, with projective bundle $q: \mathbb{P}(\mathcal{E}) \rightarrow X$. For $i=0, \ldots, n$, let

$$
\xi^{(i)}: A_{*+i-n}(X) \rightarrow A_{*}(\mathbb{P}(\mathcal{E}))
$$

be the composition of $q^{*}: A_{*+i-n}(X) \rightarrow A_{*+i}(\mathbb{P}(\mathcal{E}))$ followed by $\tilde{c}_{1}\left(O(1)_{\mathcal{E}}\right)^{i}$ : $A_{*+i}(\mathbb{P}(\mathcal{E})) \rightarrow A_{*}(\mathbb{P}(\mathcal{E}))$. Then the homomorphism

$$
\sum_{i=0}^{n-1} \xi^{(i)}: \oplus_{i=0}^{n} A_{*+i-n}(X) \rightarrow A_{*}(\mathbb{P}(\mathcal{E}))
$$

is an isomorphism.

(H). Let $E \rightarrow X$ be a vector bundle of rank $r$ over $X \in \mathbf{S c h}_{k}$, and let $p: V \rightarrow$ $X$ be an $E$-torsor (for the Zariski topology). Then $p^{*}: A_{*}(X) \rightarrow A_{*+r}(V)$ is an isomorphism.

(CD). For integers $r, N>0$, let $W=\mathbb{P}^{N} \times_{k} \ldots \times_{k} \mathbb{P}^{N}$ ( $r$ factors), and let $p_{i}: W \rightarrow \mathbb{P}^{N}$ be the $i$ th projection. Let $X_{0}, \ldots, X_{N}$ be the standard homogeneous coordinations on $\mathbb{P}^{N}$, let $n_{1}, \ldots, n_{r}$ be non-negative integers, and let $i: E \rightarrow W$ be the subscheme defined by $\prod_{i=1}^{r} p_{i}^{*}\left(X_{N}\right)^{n_{i}}=0$. Then $i_{*}: A_{*}(E) \rightarrow A_{*}(W)$ is injective.

Remarks 2.2. (1) Let $\mathbf{S m} / k \subset \mathbf{S c h}_{k}$ denote the full sub-category of smooth quasi-projective $k$-schemes. An oriented cohomology theory $A^{*}$ on $\mathbf{S m} / k$ in the sense of [7, Definition 1.1.2] is just an oriented Borel-Moore homology theory $A_{*}$, only with $\mathbf{S} \mathbf{c h}$ replaced everywhere by $\mathbf{S m} / k$, the fiber product $W$ in (BM2) is required to be in $\mathbf{S m} / k$ and the axiom (CD) omitted ( $c f$. [7, Proposition. 5.2.1]). The grading is reindexed:

$$
A^{*}(X):=A_{\operatorname{dim} X-*}(X) .
$$

$A^{*}(X)$ becomes a graded ring, with product $a \cup b:=\delta^{*}(a \times b), \delta: X \rightarrow X \times X$ the diagonal, and unit $1_{X}:=p_{X}^{*}(1), p_{X}: X \rightarrow$ pt the structure morphism. For a line bunde $L \rightarrow X$, let $c_{1}(L)=\tilde{c}_{1}(L)\left(1_{X}\right) \in A^{1}(X)$, then

$$
\tilde{c}_{1}(L)(a)=c_{1}(L) \cup a
$$

for all $a \in A^{*}(X)$.

(2) Let $f: Y \rightarrow X$ be a morphism in $\mathbf{S c h}_{k}$, with $X \in \mathbf{S m} / k$. Then $(f$,id $)$ : $Y \rightarrow X \times Y$ is a regular embedding; the pairing

$$
\begin{aligned}
A^{m}(X) \otimes A_{n}(Y) & \rightarrow A_{n-m}(Y) \\
a \otimes b & \mapsto(f, \mathrm{id})^{*}(a \times b)
\end{aligned}
$$


makes $A_{*}(Y)$ a graded $A^{*}(X)$-module (with $A_{-n}(Y)$ in degree $n$ ).

(3) Let $f: Y \rightarrow X$ be a projective morphism, $L \rightarrow X$ a line bundle, $s: X \rightarrow L$, $s^{\prime}: Y \rightarrow f^{*} L$ the zero sections. Applying (BM2) to the cartesian Tor-independent square

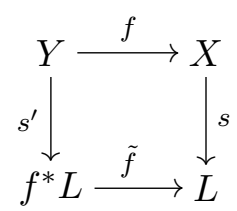

gives the projection formula for the operator $\tilde{c}_{1}(L)$ :

$$
\tilde{c}_{1}(L) \circ f_{*}=f_{*} \circ \tilde{c}_{1}\left(f^{*} L\right) .
$$

(4) For $X \in \mathbf{S c h}_{k}$, let $\mathrm{CH}_{n}(X)$ be the Chow group of dimension $n$ algebraic cycles on $X$ modulo rational equivalence, and let $\mathrm{CH}_{*}(X):=\oplus_{n} \mathrm{CH}_{n}(X)$. The Chow groups functor $X \mapsto \mathrm{CH}_{*}(X)$ is an oriented Borel-Moore homology theory on $\mathbf{S c h}_{k}$.

(5) In case we need to emphasize the particular theory, we will write $\tilde{c}_{1}^{A}(L)$ for the first Chern class operator $\tilde{c}_{1}(L): A_{*}(X) \rightarrow A_{*-1}(X)$.

One very useful consequence of the axioms is

Lemma 2.3. Let $A$ be an oriented Borel-Moore homology theory on $\mathbf{S c h}_{k}, X \in$ $\mathbf{S c h}_{k}, L \rightarrow X$ a line bundle with sheaf of sections $\mathcal{L}$. Let $s: X \rightarrow L$ a section such that the induced map $\times s: \mathcal{O}_{X} \rightarrow \mathcal{L}$ is injective, and let $i: D \rightarrow X$ be the Cartier divisor defined by $s=0$. Then

$$
\tilde{c}_{1}(L)=i_{*} i^{*}
$$

Proof. Let $s_{0}: X \rightarrow L$ be the zero section. We first show that

$$
s_{0}^{*}=s^{*}: A_{*}(L) \rightarrow A_{*-1}(X) .
$$

Indeed, we have the map $s(t): X \times \mathbb{A}^{1} \rightarrow L$ defined by $s(t)=t s+(1-t) s_{0}$, where $\mathbb{A}^{1}=$ Spec $k[t]$. Letting $i_{0}, i_{1}: X \rightarrow X \times \mathbb{A}^{1}$ be the sections with value 0,1 , respectively, it follows from the homotopy property $(\mathrm{H})$ that $i_{0}^{*}=i_{1}^{*}$, hence $s_{0}^{*}=s^{*}$, as claimed.

Now consider the cartesian square

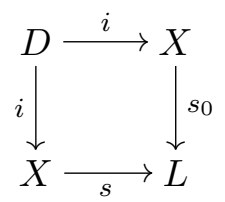


By our assumption that $\times s$ is injective, this square is Tor-independent. Clearly $s_{0}$ is projective and $s$ is an l.c.i.morphism, hence by (BM2)

$$
s^{*} s_{0 *}=i_{*} i^{*} .
$$

Since $s^{*}=s_{0}^{*}$, this shows that $i_{*} i^{*}=\tilde{c}_{1}(L)$, as desired.

The next result will be useful in proving the Whitney product formula for Chern classes of vector bundles.

Proposition 2.4. Let $X$ be in $\mathbf{S c h}_{k}$, and let $D_{1}, \ldots, D_{n}$ be effective Cartier divisors on $X$ such that for each $j=1, \ldots, n$,

$$
\operatorname{Tor}_{1}^{\mathcal{O}_{X}}\left(\otimes_{i=1}^{j-1} \mathcal{O}_{D_{i}}, \mathcal{O}_{D_{j}}\right)=0
$$

Suppose in addition that $\cap_{i=1}^{n} D_{i}=\emptyset$. Then

$$
\prod_{i=1}^{n} \tilde{c}_{1}\left(\mathcal{O}_{X}\left(D_{i}\right)\right)=0
$$

as an operator on $A_{*}(X)$.

Proof. The hypothesis on the vanishing of Tor ${ }_{1}$ implies that $D_{1} \cap \ldots \cap D_{j}$ is a Cartier divisor on $D_{1} \cap \ldots \cap D_{j-1}$ for all $j=1, \ldots, n$. Let $i^{j}: D_{1} \cap \ldots \cap D_{j} \rightarrow X$ be the inclusion. By Lemma 2.3 and induction, it follows that

$$
\prod_{i=1}^{j} \tilde{c}_{1}\left(\mathcal{O}_{X}\left(D_{i}\right)\right)\left(A_{*}(X)\right) \subset i_{*}^{j}\left(A_{*}\left(D_{1} \cap \ldots \cap D_{j}\right)\right) .
$$

for all $j$. Since $A_{*}(\emptyset)=0$, this proves the result.

For $X \in \mathbf{S c h}_{k}$, we say that $A_{*}(X)$ is generated by quasi-projective elements if $A_{*}(X)$ is generated (as a group) by elements of the form $f_{*}(a)$, for $f: Y \rightarrow X$ projective, $Y \in \mathbf{S c h}_{k}$ quasi-projective and $a \in A_{*}(Y)$. This is the case if, for example, $X$ is itself quasi-projective.

Corollary 2.5. Take $X \in \mathbf{S c h}_{k}$ for $k$ an infinite field, and let $A$ be an oriented Borel-Moore homology theory on $\mathbf{S c h}_{k}$. Suppose $A_{*}(X)$ is generated by quasiprojective elements. Then for each $a \in A_{*}(X)$, there is an integer $N$ such that, for all line bundles $L_{1}, \ldots, L_{r}$ on $X$ with $r>N, \prod_{i=1}^{r} \tilde{c}_{1}\left(L_{i}\right)(a)=0$.

Proof. Since $A_{*}(X)$ is generated by quasi-projective elements, the projection formula of Remark 2.2(4) allows us to assume that $X$ is quasi-projective. We may therefore apply Jouanolou's trick [5]: there is a vector bundle torsor $X^{\prime} \rightarrow X$ with $X^{\prime}$ affine. By $(\mathrm{H})$, we may replace $X$ with $X^{\prime}$, and assume from the start that $X=\operatorname{Spec} A$. We will show that in this case, we may take $N=\operatorname{dim} X$. Since $k$ is infinite, there are sections $s_{i}$ of $L_{i}, i=1, \ldots, r$ such that $s_{1}, \ldots s_{j}$ form 
a regular sequence in $A$ for all $j=1, \ldots N$ and $s_{1}, \ldots, s_{N+1}$ generates the unit ideal. The result thus follows from Proposition 2.4.

\section{Algebraic Cobordism}

In [7], we (that is, the author and Fabien Morel) construct the theory of algebraic cobordism, $\Omega_{*}$, and prove most of the desired properties. In [7, Sections 5 and 6], we construct pull-back maps for l.c.i.-moprhisms and show that $\Omega_{*}$ is an oriented Borel-Moore homology theory on $\mathbf{S} \mathbf{c h} k$. We give a rough sketch of the construction here.

For $X \in \mathbf{S c h}_{k}, \Omega_{n}(X)$ is generated (as an abelian group) by cobordism cycles $\left(f: Y \rightarrow X ; L_{1}, \ldots, L_{r}\right)$, where $f$ is a projective morphism, $Y \in \mathbf{S m} / k$ is irreducible of dimension $n+r$ over $k$ and the $L_{i}$ are line bundles on $Y$. We identify two cobordism cycles if they are isomorphic over $X$ or if one reorders the $L_{i}$. One imposes two relations:

(1) $\left(f: Y \rightarrow X ; L_{1}, \ldots, L_{r}\right)=0$ if there exists a smooth morphism $\pi: Y \rightarrow$ $Z$, line bundles $M_{1}, \ldots, M_{r}$ on $Z$ with $L_{i} \cong \pi^{*} M_{i}$ for $i=1, \ldots, s \leq r$ and $\operatorname{dim}_{k} Z<s$.

(2) If $s: Y \rightarrow L$ is a section with smooth divisor $i: D \rightarrow Y$ on $Y$, then we identify $\left(f: Y \rightarrow X ; L_{1}, \ldots, L_{r}, L\right)=\left(f \circ i: D \rightarrow X ; i^{*} L_{1}, \ldots, i^{*} L_{r}\right)$.

We denote the graded group so defined by $\underline{\Omega}_{*}(X)$. One defines the Chern class operators $\tilde{c}_{1}(L)$ for $L$ a line bundle on $X$ by

$$
\tilde{c}_{1}(L)\left(\left(f: Y \rightarrow X ; L_{1}, \ldots, L_{r}\right)\right):=\left(f: Y \rightarrow X ; L_{1}, \ldots, L_{r}, f^{*} L\right) .
$$

For a projective morphism $g: X \rightarrow X^{\prime}$, define

$$
g_{*}\left(f: Y \rightarrow X ; L_{1}, \ldots, L_{r}\right):=\left(g \circ f: Y \rightarrow X^{\prime} ; L_{1}, \ldots, L_{r}\right)
$$

One has as well an evident pull-back for smooth morphisms and an evident external product. Thus we have the basic data we need to define an oriented BorelMoore weak homology theory on $\mathbf{S c h}_{k}$ (except for pull-back for l.c.i.-morphisms).

The second relation gives as a special case the relation of naive cobordism, namely: Let $p: W \rightarrow X \times \mathbb{P}^{1}$ be projective with $W \in \mathbf{S m} / k$, and with $p_{2} \circ p$ smooth over a neighborhood of $\{0, \infty\}$. Let $L_{1}, \ldots, L_{r}$ be line bundles on $W$, and let $i_{0}: W_{0} \rightarrow W, i_{\infty}: W_{\infty} \rightarrow W$ be the inclusions of the fibers over $0, \infty$. Then

$$
\left(p \circ i_{0}: W_{0} \rightarrow X, i_{0}^{*} L_{1}, \ldots, i_{0}^{*} L_{r}\right)=\left(p \circ i_{\infty}: W_{\infty} \rightarrow X, i_{\infty}^{*} L_{1}, \ldots, i_{\infty}^{*} L_{r}\right)
$$

in $\underline{\Omega}_{*}(X)$. Contrary to the purely topological theory of complex cobordism, the relations (1) and (2) do not suffice to define $\Omega_{*}$. One needs to impose the formal group law. 
Recall that a (commutative, rank one) formal group law over a commutative ring $R$ is a power series $F(u, v) \in R[[u, v]]$ satisfying the formal relations of identity, commutativity and associativity:

(1) $F(u, 0)=F(0, u)=u$.

(2) $F(u, v)=F(v, u)$

(3) $F(F(u, v), w)=F(u, F(v, w))$.

There is a universal formal group law

$$
F_{\mathbb{L}}(u, v) \in \mathbb{L}[[u, v]]
$$

the coefficient ring of the universal group law $\mathbb{L}$ is the Lazard ring. $\mathbb{L}$ is constructed in the obvious way: start with the polynomial $\operatorname{ring} \mathbb{Z}\left[\left\{A_{i j}, i, j \geq 1\right\}\right]$, and form the power series

$$
\tilde{F}(u, v):=u+v+\sum_{i, j \geq 1} A_{i j} u^{i} v^{j}
$$

The relation (1) is already satisfied; relations (2) and (3) give polynomial relations on the $A_{i j}$ and $\mathbb{L}$ is the quotient of $\mathbb{Z}\left[\left\{A_{i j}\right\}\right]$ by these relations. Letting $a_{i j}$ be the image of $A_{i j}$ in $\mathbb{L}$, the universal formal group law is $F_{\mathbb{L}}(u, v):=u+v+$ $\sum_{i, j \geq 1} a_{i j} u^{i} v^{j}$. We grade $\mathbb{L}$ by giving $a_{i j}$ degree $i+j-1$. If we give $u$ and $v$ degrees -1 , this gives $F_{\mathbb{L}}(u, v)$ total degree -1 .

To construct $\Omega_{*}$, we take the functor $\mathbb{L}_{*} \otimes_{\mathbb{Z}} \underline{\Omega}_{*}$ and impose the relations:

$$
\begin{aligned}
F_{\mathbb{L}}\left(\tilde{c}_{1}(L), \tilde{c}_{1}(M)\right)\left(f: Y \rightarrow X ; L_{1}, \ldots, L_{r}\right) & \\
& =\tilde{c}_{1}(L \otimes M)\left(f: Y \rightarrow X ; L_{1}, \ldots, L_{r}\right)
\end{aligned}
$$

for each pair of line bundles $L, M$ on $X$.

One shows that $\Omega_{*}(X)$ is generated by the elementary cobordism cycles $f$ : $Y \rightarrow X$; in particular, $\Omega_{*}(X)$ is generated by quasi-projective elements for all $X \in \mathbf{S c h}_{k}$.

The construction of the pull-back for l.c.i.-morphisms is fairly technical, and is the main task of [7, Section 6]. A main result of [7] (cf. [7, Theorem 7.1.3]) is

Theorem 3.1. Assume $k$ admits resolution of singularities.

(1) Algebraic cobordism, $X \mapsto \Omega_{*}(X)$, is the universal oriented Borel-Moore homology theory on $\mathbf{S c h}_{k}$.

(2) Algebraic cobordism, considered as an oriented cohomology theory on $\mathbf{S m} / k$, is the universal oriented cohomology theory on $\mathbf{S m} / k$. 


\section{Formal Group LAWS}

As mentioned in [7, Sections 1 and 5], each oriented Borel-Moore homology theory $A$ has a formal group law $F_{A}(u, v) \in A_{*}(k)[[u, v]]$, which gives the identity of operators

$$
F_{A}\left(\tilde{c}_{1}^{A}(L), \tilde{c}_{1}^{A}(M)\right)=\tilde{c}_{1}^{A}(L \otimes M)
$$

for each pair of line bundles $L, M$ on $X \in \mathbf{S c h}_{k}$ such that $A_{*}(X)$ is generated by quasi-projective elements. For this identity to be meaningful, one needs to know that the (commuting) operators $\tilde{c}_{1}^{A}(L)$ are locally nilpotent on $A_{*}(X)$, which follows from Corollary 2.5. The existence of $F_{A}$ follows from two applications of the projective bundle formula $(\mathrm{PB})$, which gives

$$
\begin{aligned}
& A^{*}\left(\mathbb{P}^{\infty} \times \mathbb{P}^{\infty}\right):=\lim _{n, m} A^{*}\left(\mathbb{P}^{n} \times \mathbb{P}^{m}\right) \\
& \cong \lim _{n, m} A^{*}(k)[u, v] /\left(u^{n+1}, v^{m+1}\right) \\
& =A^{*}(k)[[u, v]],
\end{aligned}
$$

the isomorphism in the second line defined by sending $a u^{i} v^{j}, a \in A^{*}(k)$, to $c_{1}\left(\mathcal{O}_{\mathbb{P}^{n} \times \mathbb{P}^{m}}(i, j)\right) \cup p^{*}(a)$, where

$$
\mathcal{O}_{\mathbb{P}^{n} \times \mathbb{P}^{m}}(i, j):=p_{1}^{*} \mathcal{O}_{\mathbb{P}^{n}}(i) \otimes p_{2}^{*} \mathcal{O}_{\mathbb{P}^{m}}(j)
$$

and $p: \mathbb{P}^{n} \times \mathbb{P}^{m} \rightarrow \mathrm{pt}$ is the structure morphism. Clearly the elements $c_{1}\left(\mathcal{O}_{\mathbb{P}^{n}} \times \mathbb{P}^{m}\right.$ $(1,1)) \in A^{1}\left(\mathbb{P}^{n} \times \mathbb{P}^{m}\right)$ define an element $c_{1}(\mathcal{O}(1,1))$ in the inverse limit, so there is a uniquely defined power series $F_{A}(u, v) \in A^{*}(k)[[u, v]]$ with

$$
c_{1}(\mathcal{O}(1,1))=F_{A}\left(c_{1}(\mathcal{O}(1,0)), c_{1}(\mathcal{O}(0,1))\right) .
$$

If $X \in \mathbf{S c h}_{k}$ is affine, then every pair of line bundles $L, M$ on $X$ comes by pull-back via a map $f: X \rightarrow \mathbb{P}^{n} \times \mathbb{P}^{m}$, with $L \cong f^{*}(\mathcal{O}(1,0)), M \cong f^{*}(\mathcal{O}(0,1))$, which gives us the identity of operators

$$
\tilde{c}_{1}(L \otimes M)=F_{A}\left(\tilde{c}_{1}(L), \tilde{c}_{1}(M)\right)
$$

by using the $A^{*}\left(\mathbb{P}^{n} \times \mathbb{P}^{m}\right)$-module structure on $A_{*}(X)$ induced by $f$. Jouanolou's trick extends this to quasi-projective $X$, which in turn yields the same identity for $X$ with $A_{*}(X)$ generated by quasi-projective elements by using the projection formula for $\tilde{c}_{1}(L)$ (Remark 2.2(4)).

For each theory $A$ there is therefore a canonical graded ring homomorphism

$$
\phi_{A}: \mathbb{L}_{*} \rightarrow A_{*}(k)
$$

with $\phi_{A}\left(F_{\mathbb{L}}\right)=F_{A}$. One main result of $[7]$ is the identification of $F_{\Omega}$ with $F_{\mathbb{L}}$ :

Theorem 4.1 ([7, Theorem 4.3.7]). For a field $k$ of characteristic zero, the homomorphism $\phi_{\Omega}: \mathbb{L}_{*} \rightarrow \Omega_{*}(k)$ is an isomorphism. 
The well-known additivity of the first Chern class in the Chow ring means that $F_{\mathrm{CH}}$ is the additive group

$$
F_{\mathrm{CH}}(u, v)=u+v .
$$

Let $\phi_{+}: \mathbb{L} \rightarrow \mathbb{Z}$ be the homomorphism classifying the additive group law and let $\Omega_{*}^{+}=\Omega_{*} \otimes_{\mathbb{L}} \mathbb{Z}$. By the universal property of $\Omega_{*}$, we have the canonical morphism of oriented Borel-Morel theories

$$
\psi_{\mathrm{CH}}: \Omega^{+} \rightarrow \mathrm{CH} .
$$

Theorem 4.2 ([7, Theorem 7.1.4]). $\psi_{\mathrm{CH}}: \Omega^{+} \rightarrow \mathrm{CH}$ is an isomorphism, that is, $\mathrm{CH}$ is the universal oriented theory on $\mathbf{S c h}_{k}$ with additive formal group law.

\section{Chern Classes}

We recall the construction of Chern classes and inverse Todd classes.

Let $A_{*}$ be an oriented Borel-Moore homology theory on $\mathbf{S c h}_{k}$. For simplicity, we assume that $A_{*}(X)$ is generated by quasi-projective elements for all $X \in \mathbf{S c h}_{k}$. Using the axiom (PB), Grothendieck's construction [2] allows us to define, for each vector bundle $E \rightarrow X$, the total Chern class operator

$$
\tilde{c}_{*}(E)=\sum_{i=0}^{\mathrm{rnk} E} \tilde{c}_{i}(E),
$$

with $\tilde{c}_{i}(E): A_{n}(X) \rightarrow A_{n-i}(X)$ satisfying

(0) Given vector bundles $E \rightarrow X$ and $F \rightarrow X$ on $X \in \mathcal{V}$ one has

$$
\tilde{c}_{i}(E) \circ \tilde{c}_{j}(F)=\tilde{c}_{j}(F) \circ \tilde{c}_{i}(E)
$$

for any $(i, j)$.

(1) For any line bundle $L, \tilde{c}_{1}(L)$ agrees with the one given in axiom (PB) of definition 2.1, applied to $A_{*}$.

(2) For any l.c.i. morphism $Y \rightarrow X \in \mathbf{S c h}_{k}$, and any vector bundle $E \rightarrow X$ over $X$ one has

$$
\tilde{c}_{i}\left(f^{*} E\right) \circ f^{*}=f^{*} \circ \tilde{c}_{i}(E) .
$$

(3) If $0 \rightarrow E^{\prime} \rightarrow E \rightarrow E^{\prime \prime} \rightarrow 0$ is an exact sequence of vector bundles over $X$, then for each integer $n \geq 0$ one has the following equation in $\operatorname{End}\left(A_{*}(X)\right)$ :

$$
\tilde{c}_{n}(E)=\sum_{i=0}^{n} \tilde{c}_{i}\left(E^{\prime}\right) \tilde{c}_{n-i}\left(E^{\prime \prime}\right)
$$

(4) For any projective morphism $Y \rightarrow X$ in $\mathbf{S c h}_{k}$ and any vector bundle $E \rightarrow X$ over $X$, one has

$$
f_{*} \circ \tilde{c}_{i}\left(f^{*} E\right)=\tilde{c}_{i}(E) \circ f_{*} .
$$


Moreover, the Chern class operators are characterized by the properties (0)-(3).

Proof. For the construction, let $E \rightarrow X$ be a vector bundle of rank $r, \alpha$ an element of $A_{n}(X), \mathcal{O}(1)$ the tautological quotient line bundle on $\mathbb{P}(E), q: \mathbb{P}(E) \rightarrow X$ the structure morphism. By (PB) there are unique elements $\alpha_{i} \in A_{n-i}(X)$, $i=1, \ldots, r$, such that

$$
\tilde{c}_{1}(\mathcal{O}(1))^{r}\left(q^{*} \alpha\right)+\sum_{i=1}^{r}(-1)^{i} \tilde{c}_{1}(\mathcal{O}(1))^{r-i}\left(q^{*}\left(\alpha_{i}\right)\right)=0
$$

Setting $\tilde{c}_{i}(E)(\alpha):=\alpha_{i}$ defines the operators $\tilde{c}_{i}(E)$. The properties (0), (1), (2) and (4) follow easily from the axioms for an oriented Borel-Moore homology theory, as does the fact that (0)-(3) characterize the operators.

Grothendieck's construction of Chern classes in [2] uses the additivity of the first Chern class of line bundles with respect to tensor product, while we have only a formal group law. Because of this, we give a sketch of the proof of (3) here.

Let $\tilde{c}_{*}(E)$ denote the total Chern class operator $\sum_{i} \tilde{c}_{i}(E)$. The proof of $(3)$ uses the following lemma:

Lemma 5.1. Let $X$ be in $\mathbf{S c h}_{k}, D_{1}, \ldots, D_{n}$ effective Cartier divisors on $X$ satisfying the Tor ${ }_{1}$-vanishing condition of Proposition 2.4, and with $\cap_{i=1}^{n} D_{i}=\emptyset$. Let $L_{1}, \ldots, L_{n}$ be line bundles on $X$, and let $M_{i}=L_{i} \otimes \mathcal{O}_{X}\left(D_{i}\right), i=1, \ldots, n$. Then

$$
\prod_{i=1}^{n}\left(\tilde{c}_{1}\left(M_{i}\right)-\tilde{c}_{1}\left(L_{i}\right)\right)=0
$$

as an operator on $A_{*}(X)$.

Proof. We use the formal group law $F_{A}(u, v)=u+v+\sum_{p, q \geq 1} a_{p q} u^{p} v^{q} . M_{i}=$ $L_{i} \otimes \mathcal{O}_{X}\left(D_{i}\right)$, so

$$
\begin{aligned}
\tilde{c}_{1}\left(M_{i}\right)=F_{A}\left(\tilde{c}_{1}\left(L_{i}\right), \tilde{c}_{1}\left(\mathcal{O}_{X}\left(D_{i}\right)\right)\right) & \\
& =\tilde{c}_{1}\left(L_{i}\right)+\tilde{c}_{1}\left(\mathcal{O}_{X}\left(D_{i}\right)\right)+\sum_{p, q \geq 1} a_{p q} \tilde{c}_{1}\left(L_{i}\right)^{p} \tilde{c}_{1}\left(\mathcal{O}_{X}\left(D_{i}\right)\right)^{q} .
\end{aligned}
$$

Letting $g(u, v)=\sum_{p, q \geq 1} a_{p q} u^{p} v^{q-1}$, we thus have

$$
\tilde{c}_{1}\left(M_{i}\right)-\tilde{c}_{1}\left(L_{i}\right)=\tilde{c}_{1}\left(\mathcal{O}_{X}\left(D_{i}\right)\right) \cdot g\left(\tilde{c}_{1}\left(L_{i}\right), \tilde{c}_{1}\left(\mathcal{O}_{X}\left(D_{i}\right)\right)\right) .
$$

Since all the operators $\tilde{c}_{1}(?)$ commute, the result is a consequence of Proposition 2.4.

With these results, the proof of (3) follows the line of argument used by Grothendieck in the case of additive $c_{1}$. 
Let $\mathbf{F l}\left(E^{\prime}\right) \rightarrow X, \mathbf{F l}\left(E^{\prime \prime}\right) \rightarrow X$ be the respective full flag varieties. By the projective bundle formula, the pullback map $A_{*}(X) \rightarrow A_{*+N}\left(\mathbf{F l}\left(E^{\prime}\right) \times \mathbf{F l}\left(E^{\prime \prime}\right)\right)$ is injective, so we may assume that $E$ admits a filtration by subbundles

$$
0 \subset E_{1} \subset E_{2} \subset \ldots \subset E_{n}
$$

with $E_{i}$ of rank $i$ for each $i$, and with $E^{\prime}=E_{r}, E^{\prime \prime}=E / E_{r}$ for some $r$. Let $\operatorname{Spl}\left(E_{\bullet}\right) \rightarrow X$ be the affine-space bundle of splittings of this filtration; by the homotopy axiom $(\mathrm{H})$, we may replace $X$ with $\mathbf{S p l}\left(E_{\bullet}\right)$. Thus, we may assume that $E^{\prime}$ and $E^{\prime \prime}$ are both a direct sum of line bundles, and $E=E^{\prime} \oplus E^{\prime \prime}$. This reduces us to showing: Let $L_{1}, \ldots, L_{n}$ be line bundles on $X$. Then

$$
\tilde{c}_{*}\left(\oplus_{i=1}^{r} L_{i}\right)=\prod_{i=1}^{r}\left(1+\tilde{c}_{1}\left(L_{i}\right)\right),
$$

i.e., that $\tilde{c}_{p}\left(\oplus_{i=1}^{n} L_{i}\right)$ is the $p$ th symmetric function in the operators $\tilde{c}_{1}\left(L_{1}\right), \ldots$, $\tilde{c}_{1}\left(L_{n}\right)$.

For this, let $q: \mathbb{P} \rightarrow X$ be the projective space bundle

$$
\mathbb{P}:=\operatorname{Proj}_{\mathcal{O}_{X}}\left(\operatorname{Sym}^{*}\left(\oplus_{i=1}^{r} L_{i}\right)\right),
$$

with tautological quotient $q^{*}\left(\oplus_{i=1}^{r} L_{i}\right) \rightarrow \mathcal{O}(1)$. The composition $L_{i} \rightarrow \oplus_{i=1}^{r} L_{i} \rightarrow$ $\mathcal{O}(1)$ defines the section $s_{i}: \mathcal{O}_{\mathbb{P}} \rightarrow \mathcal{O}(1) \otimes L_{i}^{-1}$; let $D_{i}$ be the divisor of $s_{i}$. If the line bundles are all trivialized on some open $U \subset X$, then the 1-sections in $L_{1}, \ldots, L_{r}$ define the homogeneous coordinates $X_{1}, \ldots, X_{r}$ on $\mathbb{P}=\mathbb{P}_{U}^{r-1}$, and $D_{i}$ is the divisor of $X_{i}$. Thus the $D_{i}$ are all Cartier divisors, and $D_{1}, \ldots, D_{r}$ satisfy the conditions of Proposition 2.4. As $\mathcal{O}(1) \cong L_{i} \otimes \mathcal{O}_{X}\left(D_{i}\right)$, it follows by Lemma 5.1 that

$$
\prod_{i=1}^{n}\left(\tilde{c}_{1}(\mathcal{O}(1))-\tilde{c}_{1}\left(L_{i}\right)\right)=0 .
$$

From the uniqueness of the relation defining the Chern class $\tilde{c}_{p}\left(\oplus_{i=1}^{n} L_{i}\right)$, this implies that $\tilde{c}_{p}\left(\oplus_{i=1}^{n} L_{i}\right)$ is the $p$ th symmetric function in the operators $\tilde{c}_{1}\left(L_{1}\right), \ldots$, $\tilde{c}_{1}\left(L_{n}\right)$, exactly as desired.

The Chern class operators yield "fomal inverse Todd classes" as follows:

Lemma 5.2. Let $A_{*}$ be an oriented Borel-Moore homology theory on $\mathbf{S c h}_{k}$ and let $\tau=\left(\tau_{i}\right) \in \Pi_{i=0}^{\infty} A_{i}(k)$, with $\tau_{0}=1$. Suppose that $A_{*}(X)$ is generated by quasiprojective elements for all $X \in \mathbf{S c h}_{k}$. Then one can define in a unique way, for each $X \in \mathbf{S c h}_{k}$ and each vector bundle $E$ on $X$, an endomorphism (of degree zero)

$$
\operatorname{Td}_{\tau}^{-1}(E): A_{*}(X) \rightarrow A_{*}(X)
$$

such that the following holds: 
(0) Given vector bundles $E \rightarrow X$ and $F \rightarrow X$ one has

$$
\operatorname{Td}_{\tau}^{-1}(E) \circ \operatorname{Td}_{\tau}^{-1}(F)=\operatorname{Td}_{\tau}^{-1}(F) \circ \operatorname{Td}_{\tau}^{-1}(E) .
$$

(1) For a line bundle L one has:

$$
\operatorname{Td}_{\tau}^{-1}(L)=\sum_{i=0}^{\infty} \tilde{c}_{1}(L)^{i} \tau_{i}
$$

(2) For any l.c.i. morphism $Y \rightarrow X$ in $\mathbf{S c h}_{k}$, and any vector bundle $E \rightarrow X$ over $X$ one has

$$
\operatorname{Td}_{\tau}^{-1}\left(f^{*} E\right) \circ f^{*}=f^{*} \circ \operatorname{Td}_{\tau}^{-1}(E) .
$$

(3) If $0 \rightarrow E^{\prime} \rightarrow E \rightarrow E^{\prime \prime} \rightarrow 0$ is an exact sequence of vector bundles over $X$, then one has:

$$
\operatorname{Td}_{\tau}^{-1}(E)=\operatorname{Td}_{\tau}^{-1}\left(E^{\prime}\right) \circ \operatorname{Td}_{\tau}^{-1}\left(E^{\prime \prime}\right)
$$

(4) For any projective morphism $Y \rightarrow X$ in $\mathbf{S c h}_{k}$ and any vector bundle $E \rightarrow X$ over $X$, one has

$$
f_{*} \circ \mathrm{Td}_{\tau}^{-1}\left(f^{*} E\right)=\mathrm{Td}_{\tau}^{-1}(E) \circ f_{*} .
$$

Proof. Once we have the Chern class operators $\tilde{c}_{p}(E)$, the construction is just an exercise in symmetric functions. Let $\mathbb{Z}[\mathbf{t}]$ be the graded polynomial algebra over $\mathbb{Z}$ with generators $t_{1}, t_{2}, \ldots$, where $t_{n}$ has degree $n$. We set $t_{0}=1$. Introduce new variables $\xi_{1}, \xi_{2}, \ldots$ of degree -1 , and let $\mathbb{Z}[\mathbf{t}]\left[\xi_{1}, \xi_{2}, \ldots, \xi_{m}\right]$ be the bi-graded extended polynomial ring, that is, we allow infinite sums of the form $\sum_{n=0}^{\infty} Q_{n}(\mathbf{t}, \xi)$, where each $Q_{n}$ is a (usual) polynomial in the $t_{i}$ and $\xi_{j}$, homogeneous of degree $n$ in the $t_{i}$.

Let $\mathbb{Z}[\mathbf{t}]\left[\xi_{1}, \xi_{2}, \ldots\right]$ be the inverse limit

$$
\mathbb{Z}[\mathbf{t}]\left[\xi_{1}, \xi_{2}, \ldots\right]:=\lim _{m} \mathbb{Z}[\mathbf{t}]\left[\xi_{1}, \xi_{2}, \ldots, \xi_{m}\right]
$$

via the maps $\mathbb{Z}[\mathbf{t}]\left[\xi_{1}, \xi_{2}, \ldots, \xi_{m}\right] \rightarrow \mathbb{Z}[\mathbf{t}]\left[\xi_{1}, \xi_{2}, \ldots, \xi_{m-1}\right]$ sending $\xi_{m}$ to zero. The element

$$
\prod_{i=1}^{\infty} \sum_{j=0}^{\infty} t_{j} \xi_{i}^{j}
$$

is in $\mathbb{Z}[\mathbf{t}]\left[\xi_{1}, \xi_{2}, \ldots\right]$, has total degree 0 and is symmetric with respect to the $\xi$ variables. Thus, letting $\sigma_{1}(\xi), \sigma_{2}(\xi), \ldots$ be the elementary symmetric functions, with $\sigma_{m}(\xi)$ thus having degree $-m$, there are unique polynomials

$$
P_{n}\left(t_{1}, \ldots, t_{n} ; \sigma_{1}, \ldots, \sigma_{n}\right) \in \mathbb{Z}\left[t_{1}, \ldots, t_{n}, \sigma_{1}, \ldots, \sigma_{n}\right]
$$

of total degree 0 and of degree $n$ in $\mathbf{t}$ such that

$$
\prod_{i=1}^{\infty} \sum_{j=0}^{\infty} t_{j} \xi_{i}^{j}=\sum_{n} P_{n}\left(t_{1}, \ldots, t_{n} ; \sigma_{1}(\xi), \ldots, \sigma_{n}(\xi)\right) .
$$


For a vector bundle $E \rightarrow X$, define the formal operator $\operatorname{Td}_{\tau}^{-1}(E)$ by

$$
\operatorname{Td}_{\tau}^{-1}(E):=\sum_{n=0}^{\infty} P_{n}\left(\tau_{1}, \ldots, \tau_{n} ; \tilde{c}_{1}(E), \ldots, \tilde{c}_{n}(E)\right) .
$$

Note that, if $E$ is a sum of line bundles, $E=\oplus_{i=1}^{r} L_{i}$, then $\tilde{c}_{p}(E)=\sigma_{p}\left(\tilde{c}_{1}\left(L_{1}\right), \ldots\right.$, $\left.\tilde{c}_{1}\left(L_{r}\right)\right)$. This and the splitting principle shows that $E \mapsto \operatorname{Td}_{\tau}^{-1}(E)$ is (formally) multiplicative in exact sequences; Corollary 2.5 thus implies that, for each $a \in$ $A_{*}(X)$, there is an $N$ such that $P_{n}\left(\tau_{1}, \ldots, \tau_{n} ; \tilde{c}_{1}(E), \ldots, \tilde{c}_{n}(E)\right)(a)=0$ for $n>$ $N$. Thus $\operatorname{Td}_{\tau}^{-1}(E)$ makes sense as an operator of degree zero on $A_{*}(X)$ and $E \mapsto \mathrm{Td}_{\tau}^{-1}(E)$ is multiplicative (as an operator) in exact sequences.

The properties listed in the statement of the lemma follow from the analogous properties of the total Chern class operators $\tilde{c}_{*}(E)$, the splitting principle and the multiplicative property of $\mathrm{Td}_{\tau}^{-1}$.

For $X \in \mathbf{S m} / k$ and $E \rightarrow X$ a vector bundle, we write $c_{i}(E)$ for $\tilde{c}_{i}(E)\left(1_{X}\right)$ and $\operatorname{td}_{\tau}^{-1}(E)$ for $\operatorname{Td}_{\tau}^{-1}(E)\left(1_{X}\right)$.

\section{TWisting A THEORY}

Let $A_{*}$ be an oriented Borel-Moore homology theory on $\mathbf{S} \mathbf{c h}{ }_{k}$ and choose elements $\tau=\left(\tau_{i}\right) \in \prod_{i=0}^{\infty} A_{i}(k)$, with $\tau_{0}=1$. We form the twisted theory $A_{*}^{(\tau)}$ with $A_{*}^{(\tau)}(X)=A_{*}(X)$ for each $X$, with the same push-forward maps $f_{*}$ and external product $\times$, but with the pull-back $f_{(\tau)}^{*}$ (for $f: Y \rightarrow X$ an l.c.i. morphism) defined by

$$
f_{(\tau)}^{*}(x):=\operatorname{Td}_{\tau}^{-1}\left(N_{f}\right)\left(f^{*}(x)\right) .
$$

Here $N_{f} \in K_{0}(Y)$ is the formal normal bundle of $f:$ if we factor $f: Y \rightarrow X$ as a regular embedding $i: Y \rightarrow P$ followed by a smooth morphism $q: P \rightarrow X$, then

$$
N_{f}:=N_{i}-i^{*} T_{q},
$$

where $T_{q}$ is the dual of the sheaf of relative Kähler differentials $\Omega_{P / X}$.

A direct calculation verifies:

Lemma 6.1. $A_{*}^{(\tau)}$ is an oriented Borel-Moore homology theory on $\mathbf{S c h}_{k}$. The first Chern class operator for this theory, $\tilde{c}_{1}^{A^{(\tau)}}$, is given by

$$
\tilde{c}_{1}^{A^{(\tau)}}(L)=\left(\mathrm{Td}_{\tau}^{-1}\right)^{A}(L) \circ \tilde{c}_{1}^{A}(L) .
$$

Remark 6.2. If we restrict to $\mathbf{S m} / k$, all the morphisms are l.c.i.-morphisms, and $\mathrm{Td}^{-1}(E)$ is cup product with $\operatorname{td}^{-1}(E)$. This allows us twist $A$ by leaving the pull-back maps the same, and altering the projective push-forward $f_{*}$ by

$$
f_{*}^{\tau}(x):=f_{*}\left(x \cup \operatorname{td}_{\tau}^{-1}\left(N_{f}\right)\right)
$$


This defines the twisted theory $A^{\tau}$ on $\mathbf{S m} / k$. The maps

$$
\cup \operatorname{td}^{-1}\left(T_{X}\right): A^{(\tau)}(X) \rightarrow A^{\tau}(X)
$$

define an isomorphism of oriented cohomology theories. Note also that $c_{1}^{\tau}(L)$ is the inverse $\tau$-Todd class of $L, \sum_{i} \tau_{i} c_{1}(L)^{i}$.

Panin [12] has proved a very general version of the Grothendieck Riemann-Roch theorem, in the setting of oriented cohomology theories (with some additional axioms). The initial data is a map of the cohomology theories

$$
\operatorname{ch}: A \rightarrow B
$$

underlying two oriented cohomology theories $A$ and $B$, that is $\operatorname{ch}(X): A(X) \rightarrow$ $B(X)$ is a ring homomorphism for each $X \in \mathbf{S m} / k$, commuting with pull-back, but not necessarily commuting with projective push-forward. Noting that $c_{1}(L)$ is defined using both pull-back and push-forward, one can (at least partially) measure the failure of ch to commute with push-forward by comparing $\operatorname{ch}\left(c_{1}^{A}(L)\right)$ with $c_{1}^{B}(L)$. If one does this in $B\left(\mathbb{P}^{\infty}\right)=B(k)\left[[t], t=c_{1}^{B}(\mathcal{O}(1))\right.$, one has the inverse Todd genus

$$
\operatorname{Td}_{\mathrm{ch}}^{-1}(t):=\frac{\operatorname{ch}\left(c_{1}^{A}(\mathcal{O}(1))\right.}{t} .
$$

Suppose that the constant term of $\operatorname{Td}_{\mathrm{ch}}^{-1}(t)$ is 1 (one can handle the case of constant term a unit as well, but we omit this). Writing $\operatorname{Td}_{\text {ch }}^{-1}(t)=1+\sum_{i \geq 1} \tau_{i} t^{i}$, $\tau_{i} \in B(k)$, one has the twisted theory $B^{\tau}$. Since $B^{\tau}$ and $B$ have the same pullback maps, we can consider ch as a map of cohomology theories

$$
\text { ch }: A \rightarrow B^{\tau} \text {. }
$$

Panin's Riemann-Roch theorem can be interpreted in this language as stating that ch : $A \rightarrow B^{\tau}$ is a map of oriented cohomology theories, i.e., ch intertwines the push-forward maps for $A$ and for $B^{\tau}$. The explicit form of the push-forward maps for $B^{\tau}$ in terms of the inverse Todd class of the virtual normal bundle of a map $f: Y \rightarrow X$ (which is the usual Todd class of the virtual tangent bundle $\left.T_{Y}-f^{*} T_{X}\right)$ recovers the Riemann-Roch theorem in its usual form:

$$
f_{*}\left(\operatorname{ch}(x) \cup \operatorname{td}\left(T_{Y}-f^{*} T_{X}\right)\right)=\operatorname{ch}\left(f_{*} x\right) .
$$

In words: Once the theory $B$ is twisted so that ch commutes with push-forward by the zero-section $\mathbb{P}^{n} \rightarrow \mathcal{O}_{\mathbb{P} n}$ (1) (for all $n$ ), ch automatically commutes with all projective push-forward maps.

\section{SteEnRod operations}

We construct the Steenrod operations by applying the twisting operation to a polynomial extension of $\mathrm{CH}_{*} \otimes \mathbb{F}_{p}$. 
Fix a prime $p$ and let $b_{1}^{(p)}, b_{2}^{(p)}, \ldots$ be indeterminates, with $b_{n}^{(p)}$ having degree $p^{n}-1$; we set $b_{0}^{(p)}:=1$. Let $\mathbb{F}_{p}\left[b^{(p)}\right]$ be the polynomial ring on the $b_{1}^{(p)}, b_{2}^{(p)}, \ldots$ and set

$$
\begin{aligned}
& \overline{\mathrm{CH}}_{*}:=\mathrm{CH}_{*} \otimes_{\mathbb{Z}} \mathbb{F}_{p} \\
& \overline{\mathrm{CH}}\left[b^{(p)}\right]_{*}:=\mathrm{CH}_{*} \otimes_{\mathbb{Z}} \mathbb{F}_{p}\left[b^{(p)}\right] .
\end{aligned}
$$

Form the twisted theory $\overline{\mathrm{CH}}\left[b^{(p)}\right]_{*}^{\left(b^{(p)}\right)}$, i.e., we take $\tau_{p^{n}-1}=b_{n}^{(p)}$ and $\tau_{i}=0$ if $i$ is not of the form $p^{n}-1$. The universal property of $\Omega_{*}$ gives us the morphism of oriented Borel-Moore homology theories

$$
\tilde{S}^{(p)}: \Omega_{*} \rightarrow \overline{\mathrm{CH}}\left[b^{(p)}\right]_{*}^{\left(b^{(p)}\right)} .
$$

Proposition 7.1. The map $\tilde{S}^{(p)}$ descends to a morphism of oriented Borel-Moore homology theories

$$
\bar{S}^{(p)}: \overline{\mathrm{CH}}_{*} \rightarrow \overline{\mathrm{CH}}\left[b^{(p)}\right]_{*}^{\left(b^{(p)}\right)} .
$$

Proof. Since $\mathrm{CH}_{*}$ is the universal additive theory, we need only check that the formal group law for $\overline{\mathrm{CH}}\left[b^{(p)}\right]_{*}^{\left(b^{(p)}\right)}$ is additive. By Lemma 6.1 , the first Chern class for the twisted theory $\tilde{c}_{1}^{\left(b^{(p)}\right)}$ is given by

$$
\tilde{c}_{1}^{\left(b^{(p)}\right)}(L)=\sum_{n=0}^{\infty} \tilde{c}_{1}^{\mathrm{CH}}(L)^{p^{n}} b_{n}^{(p)} \quad \bmod p .
$$

Since $\tilde{c}_{1}^{\mathrm{CH}}(L \otimes M)=\tilde{c}_{1}^{\mathrm{CH}}(L)+\tilde{c}_{1}^{\mathrm{CH}}(M)$, we have

$$
\tilde{c}_{1}^{\left(b^{(p)}\right)}(L \otimes M)=\tilde{c}_{1}^{\left(b^{(p)}\right)}(L)+\tilde{c}_{1}^{\left(b^{(p)}\right)}(M)
$$

yielding the additive group law for $\overline{\mathrm{CH}}\left[b^{(p)}\right]_{*}^{\left(b^{(p)}\right)}$.

We omit the $p$ from the notation for the rest of this section. Let $R:=$ $\left(r_{1}, r_{2}, \ldots r_{n}\right)$ be a sequence of non-negative integers. Let $b^{R}:=\prod b_{i}^{r_{i}},|R|:=$ $\sum_{i} r_{i}\left(p^{i}-1\right)=\operatorname{deg}\left(b^{R}\right)$. We can thus write $\bar{S}: \mathrm{CH}_{*} \rightarrow \overline{\mathrm{CH}}[b]_{*}^{(b)}$ as

$$
\bar{S}=\sum_{R} \bar{S}_{R} \cdot b^{R}: \mathrm{CH}_{*} \rightarrow \overline{\mathrm{CH}}[b]_{*}^{(b)} .
$$

Similarly, for a vector bundle $E \rightarrow X$, we have the formal inverse Todd class $\operatorname{Td}_{(b)}^{-1}(E)$. We view $\operatorname{Td}_{(b)}^{-1}(E)$ as a "twisted total Chern class endomorphism", written as

$$
\begin{aligned}
& \operatorname{Td}_{(b)}^{-1}(E):=\sum_{R} \tilde{c}_{R}(E) b^{R} ; \\
& \tilde{c}_{R}(E): \mathrm{CH}_{*} \rightarrow \mathrm{CH}_{*-|R|} .
\end{aligned}
$$


We record the principal properties of the map $\bar{S}$ and the maps $\bar{S}_{R}$; these properties all follow immediately from the fact that $\bar{S}$ is a morphism of oriented Borel-Moore homology theories.

(1) For each $R, \bar{S}_{R}: \overline{\mathrm{CH}}_{*} \rightarrow \overline{\mathrm{CH}}_{*-R}$ is an natural transformation of functors $\mathbf{S c h}_{k}^{\prime} \rightarrow \mathbf{A b}$, i.e., for each $X \in \mathbf{S c h}_{k}, \bar{S}_{R}(X): \overline{\mathrm{CH}}_{*}(X) \rightarrow \overline{\mathrm{CH}}_{*-R}(X)$ is additive, and the maps $\bar{S}_{R}(X)$ commute with the pushforward maps $f_{*}$ for $f: X \rightarrow Y$ projective.

(2) Let $f: Y \rightarrow X$ be a l.c.i. morphism. Then

$$
\bar{S} \circ f^{*}=\operatorname{Td}_{(b)}^{-1}\left(N_{f}\right) \circ f^{*} \circ \bar{S}
$$

(3) For classes $x \in \overline{\mathrm{CH}}_{*}(X), y \in \overline{\mathrm{CH}}_{*}(Y)$,

$$
\bar{S}(x \times y)=\bar{S}(x) \times \bar{S}(y) \in \overline{\mathrm{CH}}[b]_{*}^{(b)}(X \times Y) .
$$

These properties yield the following formula for $\bar{S}_{R}$ :

Proposition 7.2. Let $Z \subset X$ be a subvariety of some $X \in \mathbf{S c h}_{k}$, let $\tilde{Z} \rightarrow Z$ be a resolution of singularities and let $f: \tilde{Z} \rightarrow X$ be the evident morphism. Then

$$
\bar{S}_{R}(1 \cdot Z)=f_{*}\left(c_{R}\left(-T_{\tilde{Z}}\right)\right)
$$

Proof. Let $p: \tilde{Z} \rightarrow$ pt be the structure morphism. $1 \cdot Z$ is clearly $f_{*}\left(1_{\tilde{Z}}\right)$. Also, $\bar{S}_{R}(\mathrm{pt})=0$ for all $R \neq \emptyset$ by reasons of degree, i.e., $\bar{S}(\mathrm{pt})=\mathrm{id}$. Thus

$$
\begin{aligned}
\bar{S}(1 \cdot Z) & =\bar{S}\left(f_{*}\left(p^{*}(1)\right)\right) \\
& =f_{*}\left(\bar{S}\left(p^{*}(1)\right)\right) \\
& =f_{*}\left(\operatorname{Td}_{(b)}^{-1}\left(N_{p}\right)\left(p^{*}(\bar{S}(1))\right)\right) \\
& =f_{*}\left(\operatorname{Td}_{(b)}^{-1}\left(N_{p}\right)\left(1_{\tilde{Z}}\right)\right) \\
& =f_{*}\left(\operatorname{td}_{(b)}^{-1}\left(-T_{\tilde{Z}}\right)\right) .
\end{aligned}
$$

Taking the coefficient of $b^{R}$ finishes the proof.

This last proposition completely describes the operations $\bar{S}_{R}$; by basic properties of Brosnan's Steenrod operations outlined in [1, Section 8], this also shows that they coincide with the Steenrod operations defined by Brosnan. Of course, Brosnan's operations have the advantage that they are defined in arbitrary characteristic. 


\section{Characteristic nUmbers}

An integral lifting of the construction of the last section gives rise to interesting characteristic numbers.

For $p: X \rightarrow$ pt smooth and projective of dimension $d$, we set $[X]:=p_{*}\left(1_{X}\right) \in$ $\Omega_{d}(k)$. By [7, Lemma 2.5.11], $\Omega_{d}(k)$ is generated by the classes $[X]$.

Fix a prime $p$, let $b_{n}=b_{n}^{(p)}$, etc. Form the twisted theory $\mathrm{CH}_{*}[b]^{(b)}$, giving us the morphism of oriented Borel-Moore theories on $\mathbf{S} \mathbf{c h}_{k}$,

$$
S: \Omega_{*} \rightarrow \mathrm{CH}[b]_{*}^{(b)},
$$

which we may write as $S=\sum_{R} S_{R} \cdot b^{R}$.

Since we are using integral coefficients rather than mod $p$ coefficients, the map $S$ will not descend to $\mathrm{CH}_{*}$, however, the fact that it does modulo $p$ has as consequence:

Lemma 8.1. Let $\Omega_{>0}(k)$ be the ideal of $\Omega_{*}(k)$ generated by elements of degree $>0$. For all $R \neq \emptyset, S_{R}\left(\Omega_{>0}(k)\right)$ is contained in $p \mathrm{CH}[b]_{*}^{(b)}(k)$.

Proof. Since $\Omega_{*}(k)=\mathbb{L}$ and $\mathrm{CH}_{*}(k)=\Omega_{*}(k) \otimes_{\mathbb{L}} \mathbb{Z}, \Omega_{>0}(k)$ is the kernel of the canonical map $\Omega_{*}(k) \rightarrow \mathrm{CH}_{*}(k)=\mathbb{Z}$. Since $S \bmod p$ factors through $\mathrm{CH}_{*}$, the result follows.

Using $1 \in \mathrm{CH}_{0}(k)$ as a generator, we have the canonical identification of $\mathrm{CH}[b]_{*}^{(b)}(k)$ with the polynomial ring $\mathbb{Z}\left[b_{1}, b_{2}, \ldots\right]$. Thus, setting $s_{R}:=\left(S_{R} / p\right)$, we have for each $R \neq \emptyset$ the well-defined homorphism

$$
s_{R}: \Omega_{|R|}(k) \rightarrow \mathbb{Z}
$$

Explicitly, Lemma 8.1 shows that, for each $R \neq \emptyset$, and each smooth projective $X$ of dimension $|R|$ over $k, p \mid \operatorname{deg}\left(c_{R}\left(-T_{X}\right)\right)$, and $s_{R}$ is the unique homomorphism with

$$
s_{R}([X])=\frac{1}{p} \cdot \operatorname{deg}\left(c_{R}\left(-T_{X}\right)\right) .
$$

The proof of these statements follows by a computation similar to that used in the proof of Proposition 7.2, and the fact that $\Omega_{*}(k)$ is generated by the classes $[X]$. We write $s_{R}(X)$ for $s_{R}([X])$.

The characteristic numbers $s_{R}$ have a nice primitivity property, modulo a certain ideal. For a $k$-scheme $X$ of finite type over $k$, we let $I(X)$ denote the ideal in $\mathbb{Z}$ generated by the field extension degrees $[k(x): k]$, as $x$ runs over the closed points of $X$. If $X$ is projective, $I(X)$ is just the image of $\mathrm{CH}_{0}(X)$ under pushforward $p_{X *}: \mathrm{CH}_{0}(X) \rightarrow \mathrm{CH}_{0}(\mathrm{pt})=\mathbb{Z}$. 
Lemma 8.2. Let $X=Y \times Z$ be a product of smooth projective varieties $Y$ and $Z$ over $k$, with $\operatorname{dim} Y>0$ and $\operatorname{dim} Z>0$. Then for all $R \neq \emptyset, s_{R}(X) \equiv 0$ $\bmod I(Z)$.

Proof. Since $S$ is a morphism of oriented Borel-Moore homology theories, $S$ has the same formal properties (7.1) as $\bar{S}$, in particular

$$
\begin{aligned}
S([X]) & =S([Y \times Z]) \\
& =S([Y] \times[Z]) \\
& =S([Y]) \times S([Z])
\end{aligned}
$$

Thus

$$
\begin{aligned}
s_{R}(X) & =\frac{1}{p} S_{R}([X]) \\
= & \sum_{\substack{R^{\prime}+R^{\prime \prime}=R \\
\left|R^{\prime}\right|=\operatorname{dim} Y,\left|R^{\prime \prime}\right|=\operatorname{dim} Z}} \frac{1}{p} S_{R^{\prime}}([Y]) \cdot S_{R^{\prime \prime}}([Z]) \\
= & \sum_{\substack{R^{\prime}+R^{\prime \prime}=R \\
\left|R^{\prime}\right|=\operatorname{dim} Y,\left|R^{\prime \prime}\right|=\operatorname{dim} Z}} s_{R^{\prime}}(Y) \cdot S_{R^{\prime \prime}}(Z) .
\end{aligned}
$$

But $S_{R^{\prime \prime}}(Z)$ is the degree of the zero-cycle $c_{R^{\prime \prime}}\left(-T_{Z}\right)$ and $s_{R^{\prime}}(Y)$ is an integer, so the last sum is $0 \bmod I(Z)$.

\section{Degree formulas}

Using the generalized degree formula of [7, Section 4.4], it is easy to show that the characteristic numbers $s_{R}$ satisfy a "degree formula". We fix a characteristic zero base field $k$ and a prime number $p$. To emphasize the dependence on the choice of $p$, we write $s_{R^{p}}(X)$ for $s_{R}(X)$ and $\left|R^{p}\right|$ for $|R|$.

Theorem 9.1. Let $f: Y \rightarrow X$ be a k-morphism of smooth projective varieties over $k$. Let $R=\left(r_{1}, \ldots, r_{n}\right)$ be a sequence of non-negative integers with $\left|R^{p}\right|=$ $\operatorname{dim} X=\operatorname{dim} Y$. Then

$$
s_{R^{p}}(Y) \equiv \operatorname{deg} f \cdot s_{R^{p}}(X) \quad \bmod I(X) .
$$

Proof. It follows from the generalized degree formula [7, Theorem 4.4.7] that there are smooth projective $k$-schemes $\tilde{Z}_{i}$, morphisms $f_{i}: \tilde{Z}_{i} \rightarrow X$ and elements $\alpha_{i} \in \Omega_{*}(k), i=1, \ldots, m$, such that

(1) $\tilde{Z}_{i} \rightarrow f_{i}\left(\tilde{Z}_{i}\right)$ is birational.

(2) $\operatorname{dim} \tilde{Z}_{i}<\operatorname{dim} X$ 
(3) $f_{*}\left(1_{Y}\right)=\operatorname{deg} f \cdot 1_{X}+\sum_{i=1}^{m} \alpha_{i} \cdot f_{i *}\left(1_{\tilde{Z}_{i}}\right)$.

Pushing forward the last identity to $\Omega_{*}(k)$ gives

$$
[Y]=\operatorname{deg} f \cdot[X]+\sum_{i} \alpha_{i} \cdot\left[\tilde{Z}_{i}\right]
$$

Since $\operatorname{dim} \tilde{Z}_{i}<\operatorname{dim} X$, it follows that $\alpha_{i}$ is in the ideal $\Omega_{*>0}(k)$ for each $i$. As $\Omega_{d}(k)$ is generated by the classes $[W]$, with $W$ smooth and projective over $k$ and $\operatorname{dim} W=d$, it follows that each $\alpha_{i}$ is a sum

$$
\alpha_{i}=\sum_{j} n_{i j}\left[W_{i j}\right]
$$

with $W_{i j}$ smooth and projective over $k, \operatorname{dim} W_{i j}>0$, and the $n_{i j}$ are integers.

If $\operatorname{dim} \tilde{Z}_{i}>0$, then it follows from Lemma 8.2 that $s_{R^{p}}\left(\alpha_{i} \cdot\left[\tilde{Z}_{i}\right]\right) \equiv 0 \bmod I\left(\tilde{Z}_{i}\right)$. Since we have the morphism $\tilde{Z}_{i} \rightarrow X, I\left(\tilde{Z}_{i}\right) \subset I(X)$. Thus

$$
s_{R^{p}}(Y) \equiv \operatorname{deg} f \cdot s_{R^{p}}(X)+\sum_{i}^{\prime} s_{R^{p}}\left(\alpha_{i} \cdot\left[\tilde{Z}_{i}\right]\right) \bmod I(X)
$$

where $\sum_{i}^{\prime}$ means the sum over all $i$ such that $\operatorname{dim} \tilde{Z}_{i}=0$.

In case $\operatorname{dim} \tilde{Z}_{i}=0$, it follows from (1) that $f_{i}: \tilde{Z}_{i} \rightarrow f_{i}\left(\tilde{Z}_{i}\right)$ is an isomorphism, and $f_{i}$ thus identifies $\tilde{Z}_{i}$ with a closed point $z_{i}$ of $X$. It thus follows that $\left[\tilde{Z}_{i}\right]=$ $\left[z_{i}\right]=\left[k\left(z_{i}\right): k\right] \cdot 1 \in \Omega_{0}(k)=\mathbb{Z}(c f .[7$, Theorem 2.5.12] $)$.

Thus

$$
s_{R^{p}}\left(\alpha_{i} \cdot\left[\tilde{Z}_{i}\right]\right)=\left[k\left(z_{i}\right): k\right] \cdot s_{R^{p}}\left(\alpha_{i}\right) \equiv 0 \bmod I(X),
$$

and we have

$$
s_{R^{p}}(Y) \equiv \operatorname{deg} f \cdot s_{R^{p}}(X) \quad \bmod I(X)
$$

as desired.

Note that the ideal $I(X)$ is a birational invariant for $X$ smooth and projective over $k$; indeed, if $U \subset X$ is open and dense, the map $z_{0}(U) \rightarrow \mathrm{CH}_{0}(X)$ is surjective, from which the birational invariance follows easily. The birational invariance of $s_{R^{p}}(X) \bmod I(X)$ follows from the degree formula:

Corollary 9.2. Let $X$ and $X^{\prime}$ be smooth projective varieties over $k$ which are birational as $k$-schemes. Then

$$
s_{R^{p}}(X) \equiv s_{R^{p}}\left(X^{\prime}\right) \quad \bmod I(X)=I\left(X^{\prime}\right)
$$

for all $R$.

Proof. Using resolution of singularities, we can assume that there is a projective birational morphism $f: X^{\prime} \rightarrow X$; since $\operatorname{deg} f=1$, the result follows from the degree formula. 


\section{Applications}

The application of degree formulas to showing "non-congruences" of correspondences was first noticed by Rost [13] in his work on generic splitting varieties, related to the proof of Bloch-Kato conjecture. Merkurjev gives a number of further applications in [10]; we give a quick sketch of Rost's basic idea (as formulated in Merkurjev's paper) and a few of the notable applications here, refering the interested reader to Merkurjev's paper for further examples.

The reader can find a treatment of Rost's applications to generic splitting varieties in [6].

To make the arguments work, we need to assume $k$ has characteristic zero; Merkurjev's treatment avoids this restriction.

Fix a prime $p$, and let $\nu_{p}$ denote the $p$-adic valuation. For each smooth projective $X$ over $k$, let $n_{X}$ be the positive generator of $I(X)$. Note that $p s_{R^{p}}(X)=$ $S_{R^{p}}([X])=\operatorname{deg} c_{R^{p}}\left(-T_{X}\right)$, hence $p s_{R^{p}}(X) \equiv 0 \bmod I(X)$.

The basic principle is

Proposition 10.1 (Theorem 7.2 of [10]). Fix a prime p. Let $X$ be a smooth projective variety of dimension $d$ and suppose there is an index $R$ such that $s_{R^{p}}(X) \not \equiv 0 \bmod I(X)$. Let $Y$ be a second smooth projective variety and let $\gamma \in \mathrm{CH}_{d}(X \times Y)$ be a correspondence. Suppose that $\operatorname{deg}_{X} \gamma$ is prime to $p$ and that $\nu_{p}\left(n_{Y}\right) \geq \nu_{p}\left(n_{X}\right)$. Then

(1) $\operatorname{dim} Y \geq \operatorname{dim} X$

(2) If $\operatorname{dim} Y=\operatorname{dim} X$, then $s_{R^{p}}([Y]) \not \equiv 0 \bmod I(Y), \nu_{p}\left(n_{Y}\right)=\nu_{p}\left(n_{X}\right)$ and there is an irreducible component $Z$ of $\gamma$ with $\operatorname{deg}_{Y} Z$ prime to $p$.

Proof. We follow Merkurjev's argument. We first prove (2). Since $\operatorname{deg}_{X} \gamma$ is prime to $p$ there is some irreducible component $Z$ of $\gamma$ with $\operatorname{deg}_{X} Z$ prime to $p$, so we may assume $\gamma=1 \cdot Z$. Take a resolution of singularities $\tilde{Z} \rightarrow Z$, giving us the diagram

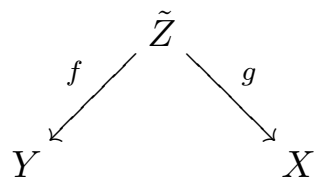

with $\operatorname{deg} g$ prime to $p$. The degree formula applied to $g$ shows that $s_{R^{p}}(\tilde{Z}) \not \equiv 0$ $\bmod I(X)$ hence

$$
s_{R^{p}}(\tilde{Z}) \not \equiv 0 \bmod I(Y) .
$$

Applying the degree formula to $f$ yields $\operatorname{deg} f \cdot s_{R^{p}}(Y) \not \equiv 0 \bmod I(Y)$. As $p s_{R^{p}}(Y) \equiv 0 \bmod I(X), \operatorname{deg} f$ must be prime to $p$ and $s_{R^{p}}(Y) \not \equiv 0 \bmod I(Y)$. 
Since $Z$ gives correspondences from $Y$ to $X$ and from $X$ to $Y$ of degree prime to $p$, we have $\nu_{p}\left(n_{X}\right)=\nu_{p}\left(n_{Y}\right)$, proving $(2)$.

For (1), suppose that $\operatorname{dim} Y<\operatorname{dim} X$, let $d=\operatorname{dim} X-\operatorname{dim} Y$ and replace $Y$ with $Y \times \mathbb{P}^{d}$, $\gamma$ with $\gamma \times 0$; this leaves $n_{Y}$ and $\operatorname{deg}_{X} \gamma$ unchanged. Clearly each irreducible component $Z$ of $\gamma$ has degree 0 over $Y$, contradicting (2).

Corollary 10.2 (Corollary 7.3 of [10]). Let $X$ be a smooth projective variety. Suppose there is an index $R$ such that $s_{R^{p}}(X) \not \equiv 0 \bmod I(X)$. If $Y$ is a smooth projective variety over $k$ with $\nu_{p}\left(n_{Y}\right) \geq \nu_{p}\left(\nu_{X}\right)$ and $\operatorname{dim} Y<\operatorname{dim} X$, then there does not exist any rational map $f: X \rightarrow Y$.

Proof. The graph of a rational map $f: X \rightarrow Y$ gives a correspondence $\gamma$ of degree 1 over $X$, contradicting (1) of Proposition 10.1.

Merkurjev notes that the proposition and its corollary imply two interesting results on the splitting of quadratic forms by the function field of quadrics. For the reader's amusement, we include the statements and arguments here.

Let $R$ be the sequence which is all zeros except for a 1 in the $n$th spot. We let $d=p^{n}-1$ and set $s_{d}:=s_{R^{p}}, S_{d}:=S_{R^{p}}$. In fact, the characteristic class $c_{R^{p}}$ corresponds to the Newton polynomial $N_{d}:=\sum_{i} \xi^{d}$. For arbitrary $d$, we have the characteristic class $c_{(d)}$ associated to $N_{d}$, and this class is additive:

$$
c_{(d)}(E \oplus F)=c_{(d)}(E) \oplus c_{(d)}(F) .
$$

Of course, only for $d=p^{n}-1$ is the value $S_{d}(X):=\operatorname{deg} c_{(d)}\left(-T_{X}\right)$ divisible by $p$. The additivity of the class $c_{(d)}$ makes for easy computations (and also gives an easy proof of the indecomposablility Lemma 8.2). For instance

Lemma 10.3. Let $X$ be a hypersurface of prime degree $p$ in $\mathbb{P}^{d+1}, d=p^{n}-1$. Then $s_{d}(X)=p^{d}-d-2$. If $p \mid n_{X}$, then $s_{d}(X) \not \equiv 0 \bmod I(X)$.

Proof. Let $i: X \rightarrow \mathbb{P}^{d+1}$ be the inclusion. We have the exact sequence

$$
0 \rightarrow T_{X} \rightarrow i^{*} T_{\mathbb{P}^{d+1}} \rightarrow \mathcal{O}_{X}(p) \rightarrow 0
$$

Applying $c_{(d)}$, and using the identity $\left[T_{\mathbb{P}^{n}}\right]=(n+1)\left[\mathcal{O}_{\mathbb{P}^{n}}(1)\right]-1$ in $K_{0}\left(\mathbb{P}^{n}\right)$ and the additivity of $c_{(d)}$ gives

$$
c_{(d)}\left(-T_{X}\right)=c_{(d)}\left(\mathcal{O}_{X}(p)\right)-(d+1) \cdot c_{(d)}\left(\mathcal{O}_{X}(1)\right) .
$$

For a line bundle $L, c_{(d)}=c_{1}(L)^{d}$, so, letting $h$ be the hyperplane class in $\mathrm{CH}^{1}(X)$,

$$
S_{d}(X)=\operatorname{deg}(p h)^{d}-(d+1) \operatorname{deg} h^{d}=p^{d+1}-(d+2) p
$$

since $h^{d}$ has degree $p$. Thus

$$
s_{d}(X)=\frac{1}{p} S_{d}(X)=p^{d}-d-2 .
$$


Since $s_{d}(X)$ is therefore congruent to $-1 \bmod p$, the proof is complete.

Here are two applications to quadratic forms.

Proposition 10.4 (Hoffmann [3, Theorem 1]). Let $X_{1}$ and $X_{2}$ be anisotropic quadrics over a field $F$. If $\operatorname{dim} X_{1} \geq 2^{n}-1$ and $X_{2}$ is isotropic over $F\left(X_{1}\right)$, then $\operatorname{dim} X_{2} \geq 2^{n}-1$.

Proof. Our argument requires $F$ to have characteristic zero. $X_{2}$ being isotropic over $F\left(X_{1}\right)$ means $X_{2}$ has an $F\left(X_{1}\right)$-rational point, which is the same as saying there is a rational map $f: X_{1} \rightarrow X_{2}$. By replacing $X_{1}$ with a general linear section, we may assume that $\operatorname{dim} X_{1}=2^{n}-1$. Since $X_{1}$ and $X_{2}$ are anisotropic, Springer's theorem [14] implies $n_{X_{1}}=n_{X_{2}}=2$.

Taking $p=2, d=2^{n}-1$, Lemma 10.3 tells us that $s_{d}\left(X_{1}\right) \not \equiv 0 \bmod I\left(X_{1}\right)$. Since $n_{X_{1}}=n_{X_{2}}$, we may apply Corollary 10.2 to show that $\operatorname{dim} X_{2} \geq 2^{n}-1$.

Proposition 10.5 (Izhboldin [4, Theorem 0.6]). Let $X_{1}$ and $X_{2}$ be anisotropic quadrics over a field $F$. If $\operatorname{dim} X_{1} \geq 2^{n}-1=\operatorname{dim} X_{2}$ and $X_{2}$ is isotropic over $F\left(X_{1}\right)$, then $X_{1}$ is isotropic over $F\left(X_{2}\right)$.

Proof. We assume as before that $F$ has characteristic zero. As in the previous proposition, we may replace $X_{1}$ with a general linear section and so can assume that $\operatorname{dim} X_{1}=2^{n}-1$. If $X_{2}$ is isotropic over $F\left(X_{1}\right)$, we have a rational map $X_{1} \rightarrow X_{2}$, i.e., a correspondence on $X_{1} \times X_{2}$ of degree 1 over $X_{1}$. Since $n_{X_{1}}=$ $n_{X_{2}}=2$ and $s_{2^{n}-1}\left(X_{1}\right) \equiv 1 \bmod 2$, Proposition 10.1(2) implies that $X_{1}$ has a 0 -cycle of odd degree over $F\left(X_{2}\right)$. By Springer's theorem [14], $X_{1}$ is isotropic over $F\left(X_{2}\right)$.

\section{REFERENCES}

[1] P. Brosnan, Steenrod operations in Chow theory, Trans. Amer. Math. Soc. 355 (2003), no. 5, 1869-1903

[2] A. Grothendieck, La théorie des classes de Chern. Bull. Soc. Math. France 86 (1958) 137154.

[3] D.W. Hoffmann, Isotropy of quadratic forms over the function field of a quadric, Math. Z. 220 (1995), no. 3, 461-476.

[4] O. Izhboldin, Motivic equivalence of quadratic forms, II, Manuscripta Math. 42 (2000), 41-42.

[5] J. P. Jouanolou, Quelques calculs en K-théorie des schémas. in Algebraic K-theory, I: Higher K-theories (Proc. Conf. Seattle Res. Center, Battelle Memorial Inst., 1972), pp. 317-335. Lecture Notes in Math., 341, Springer, Berlin, 1973.

[6] S. Joukhovitski and A. Suslin, Norm varieties, preprint, May 2005. http://www.math.uiuc.edu/K-theory/0742/

[7] M. Levine and F. Morel, Algebraic cobordism, to appear, in the Springer Monographs in Mathematics Series. 
[8] M. Levine and F. Morel, Algebraic cobordism I, preprint, Feb. 2002. http://www.math.uiuc.edu/K-theory/0547/index.html .

[9] M. Levine, Algebraic cobordism II, preprint, June 2002, http://www.math.uiuc.edu/Ktheory/0577/index.html .

[10] A. Merkurjev, Steenrod operations and degree formulas. J. Reine Angew. Math. 565 (2003), 13-26.

[11] J. Milnor, The Steenrod algebra and its dual. Ann. of Math. 67 (1958), 150171.

[12] I. Panin, Riemann-Roch theorems for oriented cohomology. In: Axiomatic, enriched and motivic homotopy theory, 261-333, NATO Sci. Ser. II Math. Phys. Chem., 131, Kluwer Acad. Publ., Dordrecht, 2004.

[13] M. Rost, Construction of splitting varieties, preprint 1998. http://www.math.uni-bielefeld.de/ rost/chain-lemma.html

[14] T.A. Springer, Sur les formes quadratiques d'indice zéro. C. R. Acad. Sci. Paris 234, (1952). 1517-1519.

Marc Levine

Northeastern University

Department of Mathematics

360 Huntington Avenue, Boston

MA 02115, USA

E-mail:marc@neu.edu 